\title{
Substantivvalenz und ihre Auffassung im Lichte neuerer Forschungsansätze
}

\section{Mojmír Muzikant}

Apart from the description of substantive valency in compliance with a six-degree model of $\mathrm{G}$. Helbig, there are efforts to capture valency related attributes of nominal heads also on the textual level and within compounds. In the present article, arguments are presented against the inclusion of the textual level into dictionary entries of valent nouns and for only limitedly taking into consideration compounds. In the second part of the article, sample entries in alphabetical order and according to the principle of word fields are presented, in the latter case without stating categorial and relational features.

Multidimensional concept of valency - specific formal features - transphrastic and intraphrastic realisation of arguments.

Neben der Beschreibung der Substantivvalenz nach dem 6-Stufen-Modell von G. Helbig gibt es auch Bemühungen, die Leerstellen von valenten Substantiven auf der Textebene sowie im Inneren von Komposita zu erfassen. Im vorliegenden Artikel werden Argumente gegen die Einbeziehung der Textebene sowie für eine Restriktion bei der Aufnahme von Komposita zusammengetragen. Im zweiten Teil des Artikels werden einige Musterlemmata angeführt, und zwar alphabetisch und nach dem Wortfeldprinzip (im zweiten Fall allerdings ohne Angabe der kategoriellen und relationalen Merkmale).

Multidimensionales Valenzkonzept - formspezifische Merkmale - transphrastische und intraphrastische Argumentrealisierung

\section{Zur Einführung}

Im vorliegenden Aufsatz liegt das Augenmerk auf Verbalsubstantiven (VS), die den Kern der valenten Substantive ausmachen. Im Laufe der Zeit sind viele Arbeiten erschienen, die sich theoretisch sowie praktisch mit der Substantivvalenz auseinander gesetzt haben. Sie verfolgen meistens das Ziel, Angaben zur Valenz und Derivation von VS systematisch festzuhalten und so die Grundlage für eine Umsetzung in Wörterbuchlemmata eines Valenzwörterbuchs zu schaffen. Eine klassische Beschreibung des Valenzverhaltens stellt G. Helbigs Valenzmodell (1992: 153-169) dar, auf dessen Grundlage u. a. das Wörterbuch zur Valenz und Distribution der Substantive von Sommerfeldt/Schreiber (1980) entstanden ist. Im Folgenden wird die Auffassung der Substantivvalenz bei Hölzner (2007) näher erläutert und an geeigneten Stellen mit derjenigen bei Helbig (1992) kontrastiert. Die Gründe für eine ausführliche Behandlung von Hölzners Publikation liegen dabei auf der Hand: Er hat in seiner Monographie nicht nur die bisherigen Ergebnisse im Bereich der Substantivvalenz kritisch ausgewertet, sondern auch eine eigene Herangehensweise an die Beschreibung von VS entwickelt. Zum Prüfstein für seinen Forschungsansatz wurde eine detaillierte Analyse von dreißig ausgewählten valenten 
Substantiven, die er unter Anwendung des COSMAS-II-Korpus vorgenommen hat. Als diskussionsbedürftig erscheint uns allerdings Hölzners Auffassung von der Argumentrealisierung innerhalb von Komposita sowie in Fällen, bei denen er den nominalen Bereich der Substantivvalenz verlässt und sich der Beschreibung der Argumentrealisierungen auf der Satz- und Textebene zuwendet. Im Anschluss an die Diskussion von Hölzners Ansatz in den Abschnitten 3 bis 6 wird anhand einiger Probeartikel der Aufbau eines Stichworts in einem vom Autor geplanten Wörterbuch zur Substantivvalenz demonstriert.

\section{Bemerkungen zum Forschungsstand}

An dieser Stelle sollen kurz einige Arbeiten besprochen werden, die entweder kontrastiv angelegt sind oder die Problematik der Substantivvalenz anhand des Tschechischen behandeln. B. Stier (1986) befasste sich in ihrer an der Universität Leipzig vorgelegten Dissertation mit VS in deutschen und französischen Texten aus dem Bereich der Presse/Publizistik. Die VS werden in reverbalisierbare und lexikalisierte VS eingeteilt. Die reverbalisierbaren VS bezeichnen einen Geschehensverlauf, die lexikalisierten können als Ergebnis, Resultat eines Geschehens gedeutet werden. Die Aktualisierung eines VS als reverbalisierbar oder lexikalisiert erfolgt häufig erst im Kontext. Der substantivierte Infinitiv wird in der vorliegenden Arbeit als ein den anderen Formen gleichwertiges VS angesehen (S. 8). Stier stellt ferner fest, dass manche Verben keine Nominalisierung zulassen (S. 31 f.). Dies betrifft einige der semantischen Untergruppen der Zustandsverben, die G. Helbig (1983) anführt. Die reverbalisierbaren VS werden unterteilt (S. 41) in satzwertige VS (als Ersatz eines Nebensatzes) und in Funktionsverbgefüge (als Prädikat eines Satzes). Die lexikalisierten VS zeichnen sich meistens durch den Verlust des Prozesscharakters aus. Stier unterteilt sie nach semantischen Gesichtspunkten in verschiedene Gruppen wie etwa Gerätebezeichnungen, Dokumente, Organisationen, Resultate mentaler bzw. kognitiver Verbalhandlungen usw. Manche Gruppen haben jedoch laut Stier das Merkmal [Prozess] noch nicht völlig verloren, sie können nicht reverbalisiert werden. Genannt seien hier: Veranstaltung (Tagung, Sitzung usw.), ferner Krankheiten, Begriffe (Wettrüsten, Rüstungswettlauf usw.) (S. 42-48). Eine Reihe von VS weist einen Doppelcharakter auf, d. h. in Abhängigkeit vom Kontext können diese Lexeme entweder als lexikalisierte oder reverbalisierbare VS aktualisiert werden (S. 48). Konkreta haben laut Stier keine Wertigkeit (S. 52).

Ferner wird in der Arbeit der Frage nachgegangen (S. 53), ob Nebensätze (NS) und satzwertige VS semantisch gleichwertig sein können. Die Autorin vertritt die Auffassung, ,dass ein Satz mit einem finiten Verb mit seiner Nominalisierung in Form einer Substantivgruppe identisch ist, wenn alle Argumente des Verbs in der Nominalisierung des Verbs aktantifiziert werden können.“ Zur Feststellung der semantischen Identität zwischen einem NS und einem VS führt Stier drei Proben durch: 1. Reverbalisierung des VS in einen Nebensatz (S. 56 f.), 2. Überführung der 
Basisproposition des Basisverbs auf das VS (S. 57 f.), 3. Vergleich der Valenz von Verb und VS. Stier fügt dabei hinzu, dass es ,im Gegensatz zum Verb beim VS generell nicht nötig ist, alle in der semantischen Mikrostruktur angelegten Argumente direkt zu aktantifizieren" (S. 61).

Im zweiten Kapitel der Dissertation (S. 67-71) werden die satzwertigen VS aus der Sicht ihrer syntaktischen Funktion untersucht. Unterschieden werden die Subjekt- und Objektpositionen des VS, wobei Stier insgesamt 15 semantische Verbgruppen anführt, die mit dem VS in den oben genannten Positionen gekoppelt werden können (VS in Subjektposition - 7 Verbgruppen, VS in Objektposition - 8 Verbgruppen). Daran schließt sich im Rahmen des 2. Kapitels die Beschreibung der Funktion der VS auf der Textebene an (S. 74-78). Eine große Rolle spielen die VS laut Stier im Bereich der Textverflechtung. Der Gebrauch der VS dient einerseits zur Textprogression, d. h. zur Überführung des Rhemas eines Satzes in das Thema des nachfolgenden Satzes, andererseits sichern die VS in Form von Kondensationen die Wiederaufnahme von Bekanntem. Für Textüberschriften ist die Ersterwähnung eines Geschehens mittels VS (Expansion) üblich. Abschließend führt die Autorin einige Beispiele an (S. 124-127), bei denen die Nominalisierung Probleme mit sich bringt.

P. Bassola (2003) legte der breiten Öffentlichkeit ein zweisprachiges Wörterbuch zur Substantivvalenz (Deutsch-Ungarisch) vor. Der Schwerpunkt der Arbeit liegt primär in der Beschreibung der syntaktischen Umgebung einer Substantivgruppe, die aus der Sicht der Wortbildung durch deverbale, deadjektivische sowie auch durch nicht abgeleitete Substantive vertreten ist. Bei der Bearbeitung der Lemmata wird von den allgemeinen theoretischen Erkenntnissen der Valenzlinguistik ausgegangen, die jedoch für den Bedarf des theoretisch nicht eingeweihten Benutzers ziemlich vereinfacht wurden. Es wird zwar mit dem Begriff „Argument“ operiert, die Argumente werden dann jedoch nur zahlenmäßig unterschieden, indem sie nummeriert werden, ohne dabei auf ihre semantisch-funktionellen Rollen einzugehen. Stattdessen werden jedem Argument schematisch Realisierungsformen zugeordnet. Die nominalen Ausdrucksvarianten werden durch Indefinitpronomina bzw. durch die Kombination ,Präposition + Indefinitpronomen' signalisiert. Die satzförmigen Ausdrucksvarianten werden durch entsprechende syndetische Mittel bzw. durch Infinitivkonstruktionen mit $z u$ angedeutet. Es muss hinzugefügt werden, dass die Valenzstrukturen der Stichwörter, aus denen eindeutig die Zahl der Argumente hervorgeht, durch Rückführung auf zugrunde liegende Verben und Adjektive in Verbindung mit einschlägigem Kopulaverb bzw. durch Einbettung in Funktionsverbgefüge anschaulich vor Augen geführt werden. Die Stufe der Ableitung der Valenzstruktur geht dem Strukturkasten, d. h. den Erweiterungsmöglichkeiten des Substantivs auf höchster Ebene durch Argumente, voraus.

In der nächsten Phase werden die Realisierungsformen der einzelnen Argumente der Reihe nach mit konkreten Beispielen belegt, und zwar in deutsch-ungarischer 
Reihengfolge. Auf die Besetzung aller Stellen wird dabei in konkreten Beispielen verzichtet. Sehr oft bleibt die Position des Genitivs in der Rolle des Agens leer, es sei denn, dass sie im Text durch vorangestelltes Possessivpronomen ausgedrückt wird. Auf der letzten Stufe werden Beispielsätze aus dem COSMAS-II-Korpus angeführt, wobei die entsprechende Nominalphrase mit Ergänzungen durch Kursivschrift hervorgehoben wird. Das vorgelegte Wörterbuch zur Substantivvalenz ist von hohem praktischem Wert, und dies nicht nur für Deutschlerner, deren Muttersprache Ungarisch ist. Die bearbeiteten Lemmata sind übersichtlich gegliedert, vollständig und verständlich dargestellt. Der Benutzer kann sicher einschätzen, dass er auch Informationen über avalente Bedeutungen und phraseologische Wendungen findet.

Auch in der Bohemistik blieben die VS nicht unbeachtet. 1980 erschien eine Studie über VS im Tschechischen von J. Novotný (1980). Die Klassifizierung der Substantive erfolgt dabei unter Heranziehung zweier Kriterien: 1) Wertigkeit, 2) Semantik. Vom Gesichtspunkt der Wertigkeit unterscheidet der Autor vier Gruppen der Geschehenssubstantive, und zwar ein-, zwei-, drei- und nullwertige. Unter dem semantischen Aspekt geht es darum, zu den einschlägigen Leerstellen der Valenzstruktur korrespondierende Komponenten der intentionalen Struktur zu finden. Berücksichtigt wird der lexikalisch-semantische Charakter des Kerns der Substantivgruppe als wichtiger Faktor bei der Herausbildung der einzelnen Valenztypen. Zur Bestimmung der Aktanten in der Ausdrucksebene verwendet Novotný der herkömmlichen Satzlehre entnommene Begriffe: Genitiv, Dativ, Instrumental, Präpositionalgefüge. Von den semantischen Rollen, die in der Abhandlung auftreten, sind folgende zu nennen: Agens mit präzisierenden Merkmalen, Patiens, Person zu deren Nutzen sich ein Geschehen vollzieht, Ursache, Träger eines Geschehens, Ziel, Resultat eines Geschehens, Circumstans, Milieu, das eine Bewegung passiert, und lokale Bedeutungen, die erfragt werden können (wohin, von wo, auf welchem Weg, wo). Als entscheidendes Zuordnungsmerkmal für VS gelten bei Novotný die grammatischen und semantischen Eigenschaften des einschlägigen VS. Dies bedeutet praktisch, dass auch nicht in Verben überführbare Substantive einbezogen werden. Die formalen Mittel, mit deren Hilfe der Übergang aus dem verbalen Bereich in den nominalen realisiert wird, lässt Novotný außer Acht. Unter der Bedeutung versteht Novotný die lexikalisch-semantische Charakteristik von Substantiven, keinesfalls die Verlaufsweise des vom Substantiv benannten Geschehens. Auf Grund einer lexikalisch-semantischen Analyse der Substantive, die sich durch die gleiche Valenz (Zahl der Aktanten) und die Übereinstimmung in Bezug auf die syntaktische und semantische Umgebung auszeichnen, gelangt Novotný zur Festsetzung eines allgemeinen, für eine bestimmte Substantivgruppe gemeinsamen Bedeutungsmerkmals. Die Aktanten (ihre Zahl, syntaktische und semantische Charakteristik) bilden bei Novotný einen festen Bestandteil der Beschreibung der VS. 
Auf die Problematik der deverbalen Substantive im Tschechischen und deren Valenzeigenschaften gehen auch Karlík und Nübler (1999) ein. Gegenstand der Erwägungen sind dabei solche VS, denen im Deutschen die Konversion schreiben $>$ das Scheiben entspricht. Die Autoren untersuchen den Transformationsvorgang „Ausgangsstruktur > Substantivgruppe mit deverbalem Substantiv als Kern" und stellen Regeln auf, die den transformationellen Wert der Valenzpositionen der zugrunde liegenden Satzstrukturen in Nominalphrasen (NP) auf syntaktischer Ebene bestimmen (S. 172-175). Die Ausgangsbasis ist auf die Satzstrukturen des Typs $S_{\text {nom }}$ $-\mathrm{VF}-\mathrm{S}_{\text {acc }}, \mathrm{S}_{\text {nom }}-\mathrm{VF}, \mathrm{S}_{\text {nom }}-\mathrm{VF}$ - andere Ergänzungen als $S_{\text {acc }}$ (Präpositivergänzung, Dativergänzung) beschränkt. Die lexikalische Semantik des Verbs wird nicht näher behandelt. Von den semantischen Rollen der Aktanten wird mit Agens und Patiens gearbeitet.

Die Aufeinanderfolge der formulierten Regeln beweist, dass die Struktur der NP VS $-S_{\text {gen }}-S_{\text {instr }}$ nicht direkt auf die aktive Satzstruktur $S_{\text {nom }}^{1}-V_{\text {Fact }}-S^{2}{ }_{\text {acc }}$ zurückgeht. Dazwischengeschaltet wird die Passivtransformation $S^{2}{ }_{\text {nom }}-V_{\text {pass }}-S_{\text {instr }}^{1}$. Die Ableitung der oben angeführten NP aus dem Passivsatz erklärt, warum die Position $S_{\text {instr }}$ "schwächer" valenzgebunden ist als die Position $S_{\text {gen }}$, der transformationell die hierarchisch höchste Position $S_{\text {nom }}$ in der zugrunde liegenden passiven Satzstruktur entspricht (S. 176). Die folgenden Beispiele zeigen, dass bei einer Reduktion der Aktanten in abgeleiteten NP mit der oben angeführten Struktur (weggelassen werden kann entweder $S_{\text {gen }}$ oder $S_{\text {instr }}$ ) der Fall nur mit der besetzten Position $S_{\text {instr }}$ ohne Textbezug mit einem Fragezeichen versehen werden muss: rozbití okna větrem ,das Zerbrechen des Fensters durch den Wind' $\times$ 'rozbití vétrem, das Zerbrechen durch den Wind' (S. 176 f.). Wichtig sind dabei zwei Feststellungen, die im Aufsatz zu verzeichnen sind: (1) Die Zahl der Valenzstellen ändert sich bei der Transposition nicht (S. 169). (2) „Alle Valenzpositionen der Ausgangsstruktur werden in der davon abgeleiteten Nominalgruppe zu potentiellen Positionen [...]“ (S. 179).

\section{Multidimensionales Valenzkonzept}

Hölzner (2007) geht von dem multidimensionalen Valenzkonzept von Jacobs (1994) aus, das er an die Bedürfnisse der Substantivvalenz (S. 111-152) anpasst. Unterschieden werden dabei vier Valenzdimensionen: die semantische Valenzdimensionen („Argumenthaftigkeit“, ARG), die Dimension „Inhaltsspezifik“ (INSP) und die syntaktische Valenzdimensionen (syntaktische „Notwendigkeit“, NOT) sowie die Dimension „Formspezifik“ (FOSP), vgl. S. 113.

Die Dimension „Argumenthaftigkeit“ bedeutet, dass der Valenzkandidat in eine vom Bezugssubstantiv ausgehende Prädikation als Argument einbezogen ist (vgl. Hölzner 2007: 114). Diese Charakteristik entspricht ungefähr der Stufe III des 6Stufen-Modells von G. Helbig. Helbig nimmt dabei an, dass eine Ebene schrittweise auf die nächsten Ebenen abgebildet wird: Semantik > Syntax > morphologische 
Form. Nach Jacobs (1994) besteht dagegen keine Abbildungsrelation zwischen verschiedenen Ebenen: Die verschiedenen Dimensionen sind voneinander unabhängig.

Die Dimension der syntaktischen Notwendigkeit tritt nach Hölzner (2007: 138) ein, wenn der Aktant (Valenzkandidat) innerhalb eines gegebenen Satzes nicht weggelassen werden kann. Das Kriterium der syntaktischen Notwendigkeit findet im herkömmlichen 6-Stufen-Modell keine Anwendung. Die Gründe liegen auf der Hand, wenn man bedenkt, dass die Aktanten bei dominierenden Substantiven bis auf Ausnahmen weglassbar sind, ohne dass die Substantivgruppe dadurch die Grammatikalität bzw. den Sinn einzubüßt. Nur im Falle von einigen nominalisierten Infinitiven wie Verlassen, Meiden, Erblicken, Feststellen oder Beherrschen und einiger Ableitungen auf -ung wie etwa Berücksichtigung lässt G. Helbig (1992: 116) auch obligatorische Valenz zu. Diese Einschätzung Helbigs wird bei Hölzner (2007: 181-205) jedoch für einige der genannten Fälle anhand von korpusgestützten Untersuchungen relativiert. Im Textkorpus findet er nämlich auch Belege ohne intraphrastisch realisiertes Objekt-Argument, wie z. B. Und die Luft in den neuen Büffet-Wagen, einer mobilen Raucherlunge, wirkt zum Schneiden - zum Meiden (S. 199).

Die Dimension der Inhaltsspezifik (INSP) umfasst bestimmte kategoriale Inhaltsmerkmale eines Valenzkandidaten, die von einem valenten Substantiv gefordert werden (vgl. Hölzner 2007: 136). Sie ist im Wesentlichen mit der IV. Stufe des klassischen Valenzmodells gleichzusetzen, auf der referentiell-semantische Merkmale der Aktanten angegeben werden (vgl. Helbig 1992: 155).

\section{Fraglichkeit der Dimension Formspezifik (FOSP)}

Eine gewisse Verlegenheit ruft die vierte Dimension hervor, die bei Hölzner (2007: 140-146) unter der Bezeichnung „Formspezifik“ (FOSP) näher erläutert wird. Wenn man bloß von Hölzners einleitender Definition ausgeht (S. 140), ${ }^{1}$ so könnte man glauben, dass diese Dimension ähnlich aufgefasst wird wie die V. Stufe bei Helbig (1992: 155), auf der die morphosyntaktische Charakteristik der Aktanten angegeben wird. Die folgenden Ausführungen Hölzners legen jedoch nahe, dass diese Valenzdimension auf völlig andere Bewertungskriterien zurückgeht. Die formspezifischen Merkmale der Valenzkandidaten werden nämlich aufgrund der eingeführten Eigenschaften der Austauschbarkeit und Vorhersagbarkeit fünffach abgestuft, und zwar auf einer Skala von FOSP 1 bis FOSP 5 (vgl. S. 143). FOSP 1 subsumiertlexemspezifischeAnschlüsse, dienichtvorhersagbar und nichtaustauschbar sind, z. B. Appetit auf; FOSP 2 wird bei mehreren möglichen lexemspezifischen Anschlüssen angesetzt, die nicht vorhersagbar und ohne Bedeutungsveränderung

1 „Ein Valenzkandidat ist formspezifisch zum Bezugssubstantiv, wenn er bestimmte Formmerkmale (z. B. den Anschluss mit einer bestimmten Präposition) aufweist, die vom Bezugssubstantiv gefordert werden." 
austauschbar sind, z. B. Vertrauen auflin/zu/Nebensatz; FOSP 3 erfasst vorhersagbare Anschlüsse, z. B. mit Dativ in instrumentaler Bedeutung; FOSP 4 bezeichnet wortklassenspezifischen Anschluss, der in hohem Maße vorhersagbar ist, z. B. das Genitivattribut; FOSP 5 schließlich liegt bei frei hinzufügbaren Anschlüssen oder transphrastisch realisierten Valenzkandidaten vor. Es ist noch hinzuzufügen, dass FOSP 1 auf der Skala als prototypischer Fall von [+FOSP] und FOSP 5 als Grenzfall zu [-FOSP] verstanden wird (s. Hölzner 2007: 143).

Aus der Einteilung der Merkmale für die Formspezifik ergeben sich nicht wenige Fragen und Einwände. Keine Bedenken gibt es wohl in Bezug auf FOSP 5, da die entsprechenden Aktantenrealisierungen keineFormmerkmaletragen, dievorhersagbar und austauschbar wären. Einwände gibt es allerdings gegen die Einbeziehung von transphrastischen "Argumentrealisierungen“ in die Substantivvalenz, die in Abschnitt 5 näher ausgeführt werden sollen. Vorbehalte sind gewiss bei FOSP 3 anzumelden, wo Hölzner (S. 145) in Wirklichkeit keine valenzgebundenen Ergänzungen, sondern nur Angaben anführt. Er versucht dabei den Terminus „Angaben“ zu vermeiden, indem er z. B. von Instrumentalbestimmungen mit der Präposition mit spricht. Nichtsdestoweniger ändert diese Bezeichnung nichts an der Tatsache, dass es sich hier um Angaben handelt, auch wenn diese aus inhaltlicher Sicht manchmal wichtiger als Ergänzungen sein können (vgl. Engel 1991: 183), weil sie das dominierende Substantiv in verschiedenartige inhaltliche Zusammenhänge einzuordnen vermögen.

Merkwürdig ist Hölzners Feststellung „[...] dass die Form des Genitivattributs nicht substantivspezifisch gefordert sein kann" (S. 145). Als Gründe für seine Behauptung führt er im Wesentlichen Folgendes an: Genitivattribute stehen in völlig unterschiedlichen Relationen zum Bezugssubstantiv, in vielen Fällen werden sie eher als Angaben eines Substantivs betrachtet, weil bei der Verwendung des Genitivattributs stets eine latente possessive Konnotation mitschwingt. Dem ist m. E. entgegenzusetzen, dass hier nicht alle Genitivattribute, sondern nur diejenigen, die bei valenten Substantiven erscheinen, behandelt werden.

Die Eigenschaft der Vorhersagbarkeit des Genitivattributs, die die Grundlage für die Einordnung in die Gruppe FOSP 4 bildet, ist mit Vorsicht zu genießen. Es gibt bekanntlich Verbalsubstantive, die entweder den Genitivus objectivus oder den Genitivus subjectivus fordern. Im Falle des Genitivus subjectivus ist die zweite Stelle einem Substantiv mit Präposition vorbehalten, das dem Präpositionalobjekt des zugrunde liegenden Verbalsatzes entspricht: Die Entscheidung des Rates über das Problem (Sommerfeldt/Schreiber 1980: 166). Möglich ist aber auch die Variante, in der statt des Genitivus subjectivus ein Genitivus objectivus steht und das Agens durch eine Präpositionalphrase mit durch angeschlossen wird: die Entscheidung des Problems durch den Rat (Sommerfeldt/Schreiber 1980: 166). Daraus ergibt sich, dass wenigstens die semantische Rolle des Genitivattributs bei demselben Bezugssubstantiv nicht immer eindeutig vorhersagbar ist. 
Abschließend stellt Hölzner (2007: 146) fest, dass der eigentliche Nutzen der vorgestellten Valenzdimensionen darin besteht, dass sie autonom und voneinander unabhängig sind. Als Beweis dafür liefert er einige auszuschließende Implikationen (S. 147 f.). Im Beispiel ARG > NOT (z. B. die Einschulung meiner Tochter) steht der Valenzkandidat Hölzner zufolge in der ARG-Relation, nicht aber in der NOTRelation zum Valenzträger. Gegen diese Auslegung ist Folgendes einzuwenden: Die Berechtigung der Dimension NOT ist m. E. umstritten, weil bei valenten Substantiven - und dies bestätigt wie bereits gesagt auch Hölzner im empirischen Teil seiner Studie (S. 159-279) - Aktanten (Valenzkandidaten) bis auf einige wenige Ausnahmen ohne Verlust der Grammatikalität weglassbar sind. In demselben Beispiel (die Einschulung meiner Tochter) sieht Hölzner (S. 147) die auszuschließende Implikation ARG > FOSP, die er folgendermaßen charakterisiert: „Hier steht der Valenzkandidat zwar in der ARG-Relation, nicht aber in der FOSP-Relation zum Valenzträger (Genitivattribute fallen unter die FOSP 4 -Relation).“ Man kann allerdings einwenden, dass Hölzner nicht auf die Frage geantwortet hat, welche von den FOSP-Abstufungen innerhalb der Skala FOSP 1 [+FOSP] und FOSP 5 [-FOSP] noch formspezifisch sind. M. E. ist nur mit vier Abstufungen zu rechnen. Die unter FOSP 3 subsumierten Beispiele könnten als frei hinzufügbare Anschlüsse klassifiziert werden (neu FOSP 4). Den so freigemachten Rang FOSP 3 könnten Genitivattribute einnehmen. Dass die Unabhängigkeit der Dimensionen nicht absolut ist, wie dies Hölzner zeigt, demonstrieren folgende Beispiele, wo sich die Dimensionen (gerade umgekehrt) addieren: ARG + INSP - Jucken der Kopfhaut (Körperteil eines Menschen), Raub eines Diamanten (Gegenstände, die einen Wert haben), ARG + FOSP - Kritik (der Presse) am eigenen Staat usw.

\section{Transphrastische Argumentrealisierungen}

Kennzeichnend für Hölzners Auffassung der Substantivvalenz ist die Unterscheidung von intraphrastischen und transphrastischen Argumentrealisierungen, wobei unter ersteren Realisierungen mit Hilfe von sprachlichen Mitteln wie Genitivattribut, Präpositionalattribut, Possessivpronomen, Infinitivgruppe, Nebensatz usw. verstanden werden, die innerhalb der NP auftreten. Transphrastische Realisierungen sind dagegen solche, die nicht unmittelbar die vom Bezugssubstantiv eröffneten Leerstellen besetzen, sondern als Partnerwörter innerhalb der entsprechenden Satzstruktur oder bloß als Elemente im weiteren Kontext auftreten.

Im Falle der Partnerwörter knüpft Hölzner an B. Sandberg (1979: 26-57) an, der in seiner Studie u. a. der Frage nachgegangen ist, welche Leerstellen Verbalsubstantive (VS) eröffnen, welche satzsemantischen Positionen (Argumentstellen) sie besetzen und mit welchen sprachlichen Mitteln diese repräsentiert werden. Sandberg untersucht ausführlich, unter welchen Bedingungen das Genitivattribut, sei es in der Funktion eines Agens oder Patiens, vorkommt. In dem Falle, dass das Genitivattribut nicht realisiert wird, ermittelt Sandberg, wie die Funktion des Agens 
bzw. Patiens anders signalisiert wird (Vorhandensein eines Partnerworts, aus dem Kontext erschließbar bzw. nicht erschließbar usw.).

Hölzner richtet sein Hauptaugenmerk auf die Argumentrealisierungen im Kontext. Anhand einer ausführlichen Analyse von Korpusbelegen ist ihm nämlich nicht entgangen, dass unbesetzte Argumentstellen innerhalb der NP oft erst aus dem Kontext zu erschließen sind. Dabei sind im Wesentlichen zwei Arten von Auslassungen zu unterscheiden (Hölzner 2007: 288): Analepse, d. h. die Auslassung bereits verbalisierter Argumente, und Katalepse, d. h. die Auslassung noch zu verbalisierender Argumente. Die analeptischen und kataleptischen Prozeduren führt Hölzner anhand von Beispieltexten vor Augen (S. 289-302). Bei der Analepse werden die fehlenden Größen eines valenten Substantivs anhand der zugrunde liegenden Satzstruktur eruiert. Im weiteren Text müssen die Attribute im Nachfeld nicht mehr ergänzt werden, weil der Rezipient die semantische Struktur der Nominalphrase (NP) schon im Vortext mental aufgebaut hat. Bei den kataleptischen Prozeduren wird auf etwas später im Text Versprachlichtes verwiesen. Die Auffassung des versprachlichten Sachverhalts als Argument wird durch das konzeptionelle Wissen des Rezipienten, durch die Aktivierung des Weltwissens, sichergestellt, d. h. mit anderen Worten erkennt der Rezipient aufgrund seines erworbenen Wissens und Erfahrungen inhaltliche Zusammenhänge und ist imstande Elemente des Textes den fehlenden Größen zuzuordnen. Es kann auch der Fall eintreten, dass die Argumentstelle im Text ungesättigt bleibt. Wir sprechen dann von einer indefiniten Auslassung.

Das Feststellen von versprachlichten Argumenten zum gegebenen valenten Substantiv auf der textuellen Ebene, d. h. im Kontext, der nicht nur die Grenze einer NP oder einer Satzstruktur überschreitet, kann sicherlich zur Beschreibung und Erklärung der Prinzipien der Textgestaltung aus Sicht der Textkontinuität beitragen. Es ist jedenfalls festzustellen, dass solche Textelemente, die als Argumente aufgrund des Sachwissens des Rezipienten dem valenten Substantiv zuzuordnen sind, primär eine kohärenzsignalisierende Funktion haben. Aus der Sicht des lexikographischen Bedarfs sind jedoch Kontextrealisierungen der Argumente mit dem Problem verbunden, wie die signalisierten Größen im Text sinnvoll und zutreffend zu erfassen und darzustellen wären. Im Hinblick auf die Dimension „Formspezifik“ ist da ja mit einer Nullrealisierung zu rechnen. (Keine formspezifischen Mittel sind zu erwarten.) Der Textaufbau, die versprachlichten Argumentgrößen, ihre Rangordnung und Wortstellung hängen in hohem Maße vom individuellen Stil eines jeden Textproduzenten ab. Für den Benutzer eines Valenzwörterbuchs ist es schließlich irrelevant, wie durch einen zielbewussten Einbau von versprachlichten Argumenten in die gegebene Textstruktur ein kohärenter Text aufgebaut wird. Aus diesen Gründen scheint es uns angebracht, auf die Erfassung der Valenzrealisierungen auf der Textebene zu verzichten und dieses Problem der Textlinguistik zu überlassen. Dieser Auffassung ist übrigens auch P. Eisenberg (2006: 5), der meint, dass Texte 
nicht Gegenstand der Grammatik im engeren Sinne (und damit auch nicht der Valenztheorie) sein können.

\section{Komposita als Domäne für intraphrastische Argumentrealisierungen}

Die Komposition ist im Deutschen eine sehr häufig vorkommende Wortbildungsart. Rein formal gesehen gibt es nur wenige Restriktionen für diesen Wortbildungstyp. Für Hölzner sind Komposita eine von mehreren Möglichkeiten der intraphrastischen Argumentrealisierung, die er zusammen mit Pronomen in die Gruppe „Sonstige“ einordnet. Dem empirischen Teil seiner Studie (Hölzner 2007: 163-279) sind quantitative Angaben zur Vorkommenshäufigkeit der einzelnen Realisierungsformen der Argumente zu entnehmen. Aus den angeführten Statistiken ist zu entnehmen, wie oft neben Komposita u. a. auch Genitiv- und Adjektivattribute zu demselben Bezugssubstantiv auftreten und welche semantischen Rollen diese sprachlichen Mittel repräsentieren. M. E. sollte man gerade diese drei oben genannten Formspezifika auch auf eventuelle semantische Verschiebungen prüfen. In diesem Zusammenhang ist angebracht, an H. Brinkmann (1962: 82) anzuknüpfen, der zwischen einem Kompositum und einer genitivischen Verknüpfung nicht selten einen gewissen Bedeutungsunterschied sieht. Dies demonstriert er am Beispiel Kleiderstoff > Stoff ihres Kleides mit folgendem erläuternden Kommentar (S. 82): „Das Substantiv im Genitiv gibt einen tatsächlichen, genau bestimmbaren Ort an (Stoff an ihrem Kleid), an dem die genannte Erscheinung ihren Platz hat. Das Vorderglied des Kompositums Kleiderstoff dagegen hat keinen konkreten Gegenstand im Auge, sondern nur eine begriffliche Unterscheidung." Der Unterschied wird abschließend wie folgt zusammengefasst: „Bei einer Zusammensetzung geht es um verschiedene Arten, bei genitivischer Fügung um einen bestimmten Platz in der Welt." Brinkmann untersucht weiter, ob das Genitivattribut zu ersetzen ist. Als Unterscheidungskriterien setzt er Umformungsproben ein. Er sagt, dass dies möglich sei, falls die genitivische Verknüpfung auf das Prädikat haben zurückgeführt werden kann, z. B. stumpfer Winkel eines Dreiecks > Das Dreieck hat einen stumpfen Winkel > stumpfwinkliges Dreieck.

\section{Entwurf einer lexikographischen Darstellung der Valenzstruktur valenter Substantive}

An die Beschreibung des Valenzverhaltens valenter Substantive ist im Prinzip von zwei Standpunkten heranzugehen. Man ist entweder bestrebt, den realen Sprachgebrauch darzustellen, d. h. im Vordergrund steht eine deskriptive Herangehensweise. Diesen Weg geht eindeutig M. Hölzner; oder im Vordergrund steht ein präskriptives Vorgehen, d. h. es wird versucht, nicht das darzustellen, „was geht“, sondern das, „was gehen soll“ (vgl. Hölzner 2007: 159). Dies bedeutet praktisch, dass alle Aktanten in einer gegebenen NP, abgesehen von konkreten Realisierungen mit 
Auslassungen, eruiert werden, die vom System her zulässig sind. Auf eventuelle Auslassungen im Text bzw. auf besondere Formspezifika der Argumente auf der textuellen Ebene wird weniger Rücksicht genommen. Nach unserer Auffassung sollte sich die lexikographische Praxis an den systemgegebenen Möglichkeiten orientieren. Transphrastische Argumentrealisierungen sollten nur im Allgemeinen (z. B. im Anhang des Wörterbuchs) in die Beschreibung der VS Eingang finden. In den einzelnen Stichwörtern werden Auslassungen von Argumenten und ihre Signalisierung durch Partnerwörter bzw. durch die Satzstruktur überschreitende Textelemente nicht illustriert. Ein solches Vorgehen steht im Übrigen im Einklang mit Helbigs grundsätzlicher Unterscheidung von substantivischer und verbaler Valenz, die laut Autor (Helbig 1992: 123) darin besteht, „dass die Substantive nicht über die Nominalphrase hinaus auf andere Einheiten des Satzes weisen, wogegen die Verben über die Determinierung der von ihnen eröffneten Leerstellen immer Klassen von Sätzen definieren."

Komposita sollten in den Aufbau des Lemmas aufgenommen werden, falls sie feste Einheiten bilden, die gegenüber der entsprechenden Nominalphrase (NP) mit dem Satelliten eine semantische Verschiebung erfahren haben. So kann z. B die NP Ausfall der Lichtmaschine (im Sinne von ,Nichtfunktionieren') u. U. in ein Kompositum überführt werden, ohne dass sich die Bedeutung ändert. Wenn aber eine NP wie Ausfall der Haare (,Herausfallen') oder Schrumpfung der Leber zu einem Kompositum umgeformt wird, entstehen medizinische Termini, die ein Symptom bzw. einen pathologischen Zustand bezeichnen: Rhythmusstörungen bis hin zu Blutdruckerhöhung, Hörverlust, Haarausfall wurden u. a. als Symptome aufgezählt. Wer unter Wassersucht, Blutarmut, Bronchitis, Grippe und Haarausfall leidet, erfährt dann dank...eine Linderung seines Leides. (COSMAS II) Dies ist auch der Grund dafür, solche Komposita in Lemmata wie Ausfall oder Schrumpfung aufzunehmen.

Die Aufzählung und Demonstrierung denotativer und formspezifischer Merkmale der Größen im Attribut sollte dank der Unterstützung durch Korpusbelege vollständig sein. Die lexikalisch-semantische Charakteristik der dominierenden Substantive wird am häufigsten mit Umschreibungen, Synonymen, Antonymen u. ä. erfasst. Die Stichwörter könnten alphabetisch oder in Wortfeldern (oder in Kongruenz- und Formklassen, gleiche Realisierung wie Verben/ unterschiedliche Realisierung) angeordnet sein.

Als Beispiel für die alphabetische Anordnung wird das Stichwort Abkühlung vorgestellt (vgl. Muzikant 2002):

\section{AвкÜHLUNG}

,Veränderung der Qualität von etwas, das Resultat des Vorgangs, die Zielgröße ist im Wortstamm enthalten (kühl) + Substantiv im Genitiv $(\mathrm{Sg})=$ Träger eines Vorgangs (Substanzen, Gegenstände, Zustände (sehr oft positive seelische Regungen, zwischenmenschliche Kontakte) 
- Abkühlung des Wasserdampf

- Abkühlung des Körpers

- Abkühlung der Bauteile

- Abkühlung der Wirtschaft

- Abkühlung des Wirtschaftsklimas

- Abkühlung der Baukonjunktur

- Abkühlung der Geschäfte

- Abkühlung der Gefühle

- Abkühlung der Beziehungen

Verwendungsbeispiele:

Trotz der leichten Abkühlung des Konjunkturklimas rechnet der Vorstand mit einem Ergebnis auf dem Niveau der ersten sechs Monate. Die Anzeichen für eine Abkühlung des Wirtschaftsklimas verstärken sich.

Substantive mit dem Merkmal [+hum] weisen in der Regel die semantische Rolle „Agens“ auf. Die Position des Genitivattributs ist für dieses relationale Merkmal gesperrt. Es kann lediglich innerhalb einer Satzstruktur als Subjekt bzw. durch das entsprechende Possessivpronomen im Attribut einer NP ausgedrückt werden:

- Damit Wasserratten im Sommer ihre Abkühlung bekommen, [....]

- Eine zweifache Mutter suchte Abkühlung im nahen Stubenbergsee [...] usw.

Anm.: Statt der Substanz, die als satzförmige Materie [Luft] mitgedacht wird, werden in der Regel Mengenbestimmungen angegeben:

- Abkühlung bis auf $3 \mathrm{Grad}$

- Abkühlung bis $1 \mathrm{Grad}$

- Abkühlung auf $8 \mathrm{Grad}$

- Abkühlung auf Werte um 8 Grad

Verwendungsbeispiel:

Am Wochenende wird es zwar eine vorübergehende Abkühlung geben, doch schon am Sonntag Abend eine deutliche Abkühlung bis zu zehn Grad.

\section{Einordnung nach dem Wortfeldprinzip}

Aus pragmatischer Sicht ist die Angabe von weiteren Kollokationsmöglichkeiten zu erwägen, die zwar den Valenzrahmen überschreiten, dafür aber in vielen Fällen nichtvalenzgebundene Begleiter erfassen, die im Text wichtiger als die Ergänzungen sein können. Die Einordnung der VS nach dem Wortfeldprinzip könnte wiederum die Bedeutungsnuancen zwischen den einzelnen Gliedern des gegebenen lexikalischen Mikrosystems durch festgestellte Differenzierungsseme besser und zutreffender 
erfassen. Für die Demonstration haben wir VS mit dem gemeinsamen Merkmal [+Besitz-/Verfügungswechsel] ausgewählt. (vgl. Muzikant 2002) Auf die Aufzählung der kategoriellen und relationalen Merkmale wird in dieser Phase verzichtet:

I. Etwas geht in den Besitz oder in die Verfügungsgewalt des Agens über:

- Annahme des Wechsels durch den Geschäftsführer

- Erhalt der Sendung durch den Empfänger

II. Durch eine konkrete und zielbewusste Tätigkeit, die in der Regel in der Wurzel des betreffenden Bezugsnomens genannt wird (in der Bedeutung ,etwas gewinnen, erlangen') geht etwas in den Besitz oder in die Verfügungsgewalt des Agens über:

- Erringung der absoluten Mehrheit durch die Partei

- Erstreitung der Gleichberechtigung durch die Opposition

III. Etwas geht in den Besitz oder in die Verfügungsgewalt des Agens im amtlichen Auftrag über:

- Verstaatlichung der Banken durch die Regierung

- Aberkennung der WM-Goldmedaille durch den Ausschuss

IV. Etwas geht in den Besitz oder in die Verfügungsgewalt des Agens über, nicht im Einklang mit den bestehenden Verhaltensnormen (oft ohne Wissen des ursprünglichen Besitzers):

- Unterschlagung der Mitgliedsbeiträge durch den Kassierer

- Veruntreuung des Reservefonds durch den Bänker

V. Etwas geht in den Besitz oder in die Verfügungsgewalt des Agens über, unter Gewaltanwendung:

- Erbeutung der Ausrüstung durch die anrückende Armee

- Besetzung des Südlibanons durch israelische Truppen

VI. Etwas geht in den Besitz oder in die Verfügungsgewalt des Agens über, gegen einen Ersatzwert (Finanzmittel):

- Einkauf von Obst und Gemüse durch die Wirtin

- Anschaffung zusätzlicher Hardware durch die Firma

VII. Etwas geht in die Verfügungsgewalt des Agens über, gegen einen Ersatzwert (Geld), (in Form eines Vertrags), in vereinbarten Raten, für eine bestimmte Zeit:

- Chartern eines Helikopters durch den Geschäftsmann

- Leasen eines Autos durch den Touristen 
VIII. Das Agens gibt etwas ab:

- Lieferung von Lebensmitteln an den Haushalt durch die Firma

- Übergabe der Abrechnungsunterlagen an die Verrechnungsstelle durch den Arzt

IX. Das Agens gibt etwas ab, für immer und ohne einen Ersatzwert

- Bescherung der Kinder mit Gaben durch den Nikolaus

- Schenkung des Nachlasses durch die Familie an die Stiftung für Photographie

X. Das Agens gibt etwas ab, für eine bestimmte Zeit, die a) von der Einhaltung gewisser Bedingungen vonseiten des Empfängers abhängt oder b) gegen Gebühr oder gegen das Versprechen der Rückgabe befristet ist:

- Verleihung der Goldenen Ehrenmedaille an die Künstlerin durch die Jury - Verleih der Langlaufausrüstung an die Winterurlauber durch den Skiverband

XI. Das Agens gibt Geld zu einem bestimmten Zweck ab:

- Begleichung der Rechnung durch den Gast

- Erstattung der Löhne durch die Regierung an die ehemaligen Zwangsarbeiter

XII. Das Agens gibt Geld ab als Entschädigung für eine geleistete Arbeit (in Form von Lohn):

- Entlohnung der Mitarbeiter durch die Firma

- Bevorschussung der Gehälter durch die Verbandsgemeinden

XIII. Das Agens gibt etwas ab und dafür erhält er als Ersatzwert etwas anderes (dies geschieht nach gemeinsamer Absprache beider Parteien):

- Tausch von Grundstücken durch die beteiligten Grundstückbesitzer

- Umtausch von Schilling in Euro durch Touristen

XIV. Tausch eines Gegenstands für Geld nach gegenseitiger Absprache beider Parteien, wobei der Gegenstand als Pfand dient, die Transaktion ist zeitweilig und reversibel. Es gibt zwei Verben mit dieser lexikalischsemantischen Charakteristik, die folgende Sichtweisen des Geschehens in der Ausdrucksstruktur reflektieren:

a) Das Agens ist derjenige, der ein Objekt zur Verfügung stellt und Geldmittel dafür erhält:

- Verpfändung der Aktien durch das Unternehmen an die Bank Austria 
b) Das Agens ist derjenige, der Geld zur Verfügung stellt und dafür einen Gegenstand zur Aufbewahrung bekommt:

- Beleihung von Autos durch die Bank

XV. Das Agens nimmt Geld als Ersatz für das Abgegebene entgegen:

- Verkauf von Waffen und Treibstoff an die Unita-Rebellen durch die Rüstungsfirmen

- Versteigerung einer Privatsammlung impressionistischer Kunstwerke durch das Auktionshaus

Im folgenden Beleg sind beide Seiten, d. h. sowohl der Käufer als auch der Verkäufer -enthalten:

- Handel mit Haifisch-Flossen

- Handel mit Elefanten

Das Agens wird entweder im Genitiv oder mit den Präpositionalfügungen ,zwischen, unter + Substantiv` ausgedrückt, falls Partner bezeichnet werden sollen:

- Handel Indiens mit Elfenbein

- Handel zwischen Russland und den Vereinigten Staaten mit verflüssigtem Erdgas

- Handel unter den EU-Staaten mit landwirtschaftlichen Erzeugnissen

XVI. Das Agens nimmt Geld als Ersatz für das Abgegebene entgegen, und zwar für eine vereinbarte Zeit und in wiederholten Zeitabständen, Gegenstand des Verfügungswechsels ist ein Grundstück oder etwas auf ihm Erbautes:

- Verpachtung der Hochwildjagd an den Pächter durch den Verpächter

- Vermietung der Wohnung an den Ausländer durch den Hausbesitzer

\section{Fazit}

In den Ausführungen oben wurde Folgendes gezeigt: Transphrastische Realisierungen von Argumenten, wie sie Hölzner (2007) annimmt, sind u. E. bei der Erarbeitung eines Valenzwörterbuchs nicht maßgeblich. Die Einbeziehung von Argumentrealisierungen auf der Textebene oder innerhalb der Satzstruktur (aber außerhalb der Nominalphrase) würde den Aufbau des nominalen Lemmas aus vielen Gründen (Überbelastung durch unnötige Informationen und dadurch entstandene Unübersichtlichkeit für den Benutzer sowie die Bandbreite der zu berücksichtigenden Merkmale) beeinträchtigen. Komposita als Konkurrenzformen zu NPs werden nur dann im Wörterbuch berücksichtigt, wenn sie eine semantische Verschiebung gegenüber den entsprechenden NPs aufweisen.

Die vorgeschlagene Einordnung der VS nach dem Wortfeldprinzip reflektiert lediglich die Bedeutungsunterschiede innerhalb eines Mikrosystems [+Besitz-/ 
Verfügungswechsel], die anhand des Vergleichs mit Hilfe von Differenzierungssemen festgestellt werden. Eine vollständige Beschreibung der Nominalphrasen (NP), deren dominierende Komponente das gemeinsame Merkmal [+Besitz-/Verfügungswechsel] trägt, erfordert außerdem die Festlegung der Anzahl der Argumentstellen, Angaben zu ihrer Realisierung auf der Ausdrucksebene sowie die funktional-semantische und denotativ-semantische Bestimmung der Aktanten, vor allem aber die Ermittlung der Restriktionen für deren lexikalisch-semantische Repräsentation. Auf die Berücksichtigung von Angaben wird verzichtet, denn dadurch würden die Lexikoneinträge ins Uferlose anschwellen.

\section{Literaturverzeichnis}

Bassola, Peter (2003): Deutsch-Ungarisches Wörterbuch zur Substantivvalenz. Szeged, Grimm. Brinkman, Hennig (1962): Die deutsche Sprache. Gestalt und Leistung. Düsseldorf, Schwann. Eisenberg, Peter (2006): Grundriss der deutschen Grammatik. Bd. 1: Das Wort. 3. Aufl. Stuttgart und Weimar, Metzler.

Engel, Ulrich (1991): Deutsche Grammatik. Heidelberg, Groos.

Helbig, Gerhard (1992): Probleme der Valenz- und Kasustheorie. Tübingen, Niemeyer.

Helbig, Gerhard (1983): Studien zur deutschen Syntax. Bd. 1. Leipzig, Enzyklopädie.

Hölzner, Matthias (2007): Substantivvalenz. Korpusgestützte Untersuchungen zu Argumentrealisierungen deutscher Substantive. Tübingen, Niemeyer.

Jacobs, Joachim (1994): Kontra Valenz. Reihe Fokus - Linguistisch-Philologische Studien, Bd. 12. Trier, Wissenschaftlicher Verlag.

Karlík, Petr / Nübler, Norbert (1999): Substantivische Nominalisierungen im Tschechischen. In: Zeitschrift für Slavistik 44. S. 167-180.

Muzikant, Mojmír (2002): Die Geschehenssubstantive des Deutschen. Vorstudien zu einem kleinen Valenzlexikon. Brno, Masarykova univerzita.

Novotný, Jiř́ (1980): Valence dějových substantiv v češtině. In: Sbornik Pedagogické fakulty $v$ Ústí n. Labem, bohemistická rada. S. 6-131.

Sandberg, Bengt (1979): Zur Repräsentation, Besetzung und Funktion einiger zentraler Leerstellen bei Substantiven. Acta Universitatis Gothoburgensis. Göteborg, Universität.

Sommerfeldt, Karl-Ernst / Schreiber, Helmut (1980): Wörterbuch zur Valenz und Distribution der Substantive. Leipzig, VEB Bibliographisches Institut.

Stier, Bettina (1986): Zum Verbalsubstantiv in ausgewählten deutschen und französischen Texten aus dem Bereich der Presse/Publizistik bzw. Wissenschaft/Technik. Diss. A. Leipzig, Universität. 